\title{
FLEXARRAY: RANDOM PHASED ARRAY LAYOUTS FOR ANALYTICAL SPATIAL FILTERING
}

\author{
Paul Hurley ${ }^{*}$, Matthieu Simeoni ${ }^{*, \dagger}$ \\ * IBM Zurich Research Laboratory, CH-8803 Rüschlikon, Switzerland \\ † École Polytechnique Fédérale de Lausanne (EPFL), CH-1015 Lausanne, Switzerland
}

\begin{abstract}
We propose a method for designing phased-arrays according to a given, analytically-specified, target beamshape. Building on the flexibeam framework, antenna locations are sampled from a probabilistic density function. Naturally scalable with the number of antennas, it is also computationally efficient and numerically stable, as it relies on analytical derivation.

We prove that, under mild conditions, the achieved beamshapes converge uniformly to the target beamshapes as the number of antennas increases. We illustrate the technique through a number of examples. For instance, by use of the Laplace filter, beams with extremely fast decay away from the centre of focus are achieved.

Some macroscopic observations result. We observe that matched beamforming weights may, for a given layout, achieve beamshapes targeting regions, rather than isolated directions as commonly believed. Additionally, the convergence analysis can be used to forecast the growth of future large phased arrays such as the Square Kilometre Array (SKA).
\end{abstract}

Index Terms-Beamforming, array signal processing, array design, spatial filtering

\section{INTRODUCTION}

Beamforming combines networks of antennas or sensors coherently so as to achieve a beamshape with desirable properties such as high directivity, reduced side-lobes, and improved SNR [1, 2, 3, 3 , 5].

It is often thought of as steering the array to focus on a particular point. This, however, has a number of drawbacks. It cannot adjust for small errors in direction of interest estimates, nor cope well with moving devices. Additionally, often a region or regions is what is desired, to scan the sky in radio astronomy or an organ in ultrasound.

As such, the flexibeam framework [6] was developed to, in a data independent fashion, determine beamforming weights so as to target a general spatial region. The framework takes as input fixed antenna locations, and approximates the target beamshapes.

The present work, flexarray, takes the alternate view. The beamforming weights are of unit magnitude, and only altered to steer the array in a certain direction. An optimal layout is determined by sampling antenna locations from a probabilistic density function, that itself is derived from the target filter.

As we will show, under mild conditions, the empirical beamshape will converge uniformly to the target as the number of antennas increases. The procedure is numerically stable, computationally efficient and versatile, offering the analyst a very powerful tool for designing beamshapes, as well as insight into how an array will scale with ever increasing numbers of antennas. In contrast to what is commonly thought, we observe that matched beamforming weights can lead to beamshapes focused on a region rather than a point.

As the beamforming weights all have unit modulus, this translates into a delay, allowing existing hardware to be used to achieve a given beam, and has the advantage of treating noise uniformly across antennas. In contrast, flexibeam modifies the magnitude of the weights at the cost of non-uniform noise distribution, but with the advantage of re-configurable beamshapes.

For simplicity in explanation, the paper considers circularly symmetric filters and 2D beamforming.

\subsection{Relation to prior work}

Phased array layouts and beamforming weights are usually chosen so as to minimise a certain objective function [7, 1] which is a linear combination of conflicting metrics, capturing some key beamshape properties: directivity, side-lobe level, main-lobe beam-width and/or signal-to-noise ratio [8, 7, 1]. For example, the Dolph-Chebyshev design [1] has been shown to achieve the narrowest beam-width for a given sidelobe level. Such design techniques were originally restricted to regular arrays [1, 9], and have recently been extended to irregular, hierarchical or sparse arrays [7] 10], more cost efficient layouts for very large arrays. Sometimes, phased arrays are also optimised for the very specific application for which they are being used. For example in radio astronomy, various layouts (T-shape, Y-shape, Reuleaux triangle,...) have been proposed to optimise the point spread function of the telescope [11, 10].

The work presented here has a radically different take on the design problem. We directly specify the radiation patterns analytically, permitting us to control much more precisely its shape. Flexibeam [6] achieved this, for a fixed layout, through modulation of both the phase and amplitude of the beamforming weights. In contrast, flexarray optimises the layout while using weights of unit amplitude, hence avoiding non-uniform noise perturbation.

\section{PRELIMINARIES}

For simplicity, we concentrate on beamforming from the receive perspective, but the quantities and methods presented in the subsequent sections remain valid in the transmit case.

\subsection{Beamforming}

Consider an array of $N$ receiving antennas, which, without loss of generality, have unit gains and an omni-directional field of view. Beamforming in mathematical terms translates into a linear com- 
bination of the antenna signals $x_{i}(t)$ :

$$
y(t)=\sum_{i=1}^{N} w_{i}^{*} x_{i}(t),
$$

where $w_{i} \in \mathbb{C}$ are beamforming coefficients.

Under the non-limiting assumptions of far-field and narrowband signals,

$$
y(t)=\int_{0}^{2 \pi} s(t, \theta) b^{*}(\theta) d \theta+\sum_{i=1}^{N} w_{i}^{*} n_{i}(t) .
$$

The function $b(\theta)=\sum_{i=1}^{N} w_{i} e^{j 2 \pi p_{i} \cos \left(\theta-\phi_{i}\right)}$ is called the beamshape of the beamformed antenna array. It describes the sensitivity of the antenna array relative to various directions $\theta$, and hence acts as an angular filter.

\subsection{Beamforming with Flexibeam}

Recently, a new framework called flexibeam was proposed in [12], permitting to achieve a wide range of such analytically-specified beamshapes. In this section we briefly recall some important notions and notations borrowed from this framework. Flexibeam's main departure from the state of the art was to consider a notional continuous field of antennas, with an associated beamforming function $\omega: \mathbb{R}^{2} \rightarrow \mathbb{C}$, describing the gains and delays to be applied at each location $(p, \phi) \in \mathbb{R}_{+} \times[0,2 \pi]$ so as to achieve an analytically specified target beamshape $\hat{\omega}:[0,2 \pi] \rightarrow \mathbb{C}:$

$$
\hat{\omega}(\theta)=\int_{0}^{\infty} \int_{0}^{2 \pi} \omega(p, \phi) e^{j 2 \pi p \cos (\theta-\phi)} p d \phi d p .
$$

The beamforming function can be computed as

$$
\omega(p, \phi)=\int_{0}^{\infty} \int_{0}^{2 \pi} \hat{\omega}_{e}(r, \theta) e^{-j 2 \pi r p \cos (\theta-\phi)} r d \theta d r
$$

where $\hat{\omega}_{e}: \mathbb{R}^{2} \rightarrow \mathbb{C}$ is called the extended filter, and such that $\hat{\omega}_{e}(1, \theta)=\hat{\omega}(\theta)$. For $N$ given antenna locations, the corresponding beamforming weights $w_{i} \in \mathbb{C}$ are then obtained by sampling $\omega$

$$
w_{i}=\frac{\omega\left(p_{i}, \phi_{i}\right)}{\beta}, \quad i=1, \ldots N
$$

where $\beta$ is a normalising constant to avoid noise magnification. The resulting beamshape then approximates the target beamshape, with accuracy dependant on the specific layout under consideration.

\section{FLEXARRAY}

In this section, we propose a framework called flexarray to generate random layouts so as to approximate analytically-specified target beamshapes. To do so, we leverage the flexibeam framework and construct a probability density function from the beamforming function. In the specific case of circularly symmetric extended filters, we link this density function to the Hankel transform of order zero of the extended filter. We finally show that the empirical beamshapes obtained when sampling this density converge uniformly almost surely to the target beamshape as the number of antennas grows to infinity. The rate of convergence is assessed through simulation.

\subsection{Beamforming with Circularly Symmetric Extended Filters}

This paper is concerned with extended filters that are real and, when translated to the origin, circularly symmetric, namely:

$$
\hat{\omega}_{e}(\boldsymbol{r})=\hat{g}\left(\left\|\boldsymbol{r}-\boldsymbol{r}_{0}\right\|\right), \quad \forall \boldsymbol{r} \in \mathbb{R}^{2},
$$

where $\hat{g}$ is a function defined over $\mathbb{R}_{+}$, and $\boldsymbol{r}_{0}=\left(\cos \theta_{0}, \sin \theta_{0}\right) \in$ $\mathbb{S}^{1}$ is the focus direction. Eq. (4) shows that the beamforming function is the 2D Fourier transform of the target filter. Leveraging the shift property, we can hence express the beamforming function in terms of the zeroth order Hankel transform of $\hat{g}$ :

$$
\begin{aligned}
\omega(p, \phi) & =e^{-j 2 \pi p \cos \left(\phi-\theta_{0}\right)} \int_{0}^{\infty} \int_{0}^{2 \pi} r \hat{g}(r) e^{-j 2 \pi r p \cos (\theta-\phi)} d \theta d r \\
& =e^{-j 2 \pi p \cos \left(\phi-\theta_{0}\right)}\left[2 \pi \int_{0}^{\infty} r \hat{g}(r) J_{0}(2 \pi r p) d r\right] \\
& =e^{-j 2 \pi p \cos \left(\phi-\theta_{0}\right)} g(p)
\end{aligned}
$$

where $J_{0}$ is the zeroth order Bessel function of the first kind. Then,

$$
g(p)=2 \pi \int_{0}^{\infty} r \hat{g}(r) J_{0}(2 \pi r p) d r
$$

which is the Hankel transform of order zero of $\hat{g}$.

\subsection{Beamforming in a Probabilistic Setup}

Assuming that the flexibeam beamforming function $\omega \in \mathcal{L}_{1}\left(\mathbb{R}^{2}\right)$, its relationship to the target beamshape from Eq. (4) can be re-written using Eq. [5], as

$$
\begin{gathered}
\hat{\omega}(\theta)=\int_{0}^{\infty} \int_{0}^{2 \pi}\left[e^{-j 2 \pi p \cos \left(\phi-\theta_{0}\right)} g(p)\right] e^{j 2 \pi p \cos (\theta-\phi)} p d \phi d p \\
=\int_{0}^{\infty} \int_{0}^{2 \pi}\|g\|_{1} \sigma_{g}(p) e^{-j 2 \pi p \cos \left(\phi-\theta_{0}\right)} \ldots \\
\cdots e^{j 2 \pi p \cos (\theta-\phi)} f_{b}(p, \phi) p d \phi d p,
\end{gathered}
$$

where $\|g\|_{1}=\int_{0}^{\infty}|g(p)| d p$ and $\sigma_{g}(p)=\operatorname{sign}(g(p))$. Now, $f_{b}$ is a probability density function, which we call the beamforming density function, and in polar coordinates is defined as

$$
f_{b}(p, \phi)=\frac{|g(p)|}{\|g\|_{1}}, \quad \forall p \in \mathbb{R}_{+} .
$$

Let $\boldsymbol{P}: \Omega \rightarrow \mathbb{R}^{2}$ be a random vector with polar coordinates $(P, \Phi)$ and probability density function $f_{b}$. Eq. (7) is then

$$
\hat{\omega}(\boldsymbol{r})=\alpha \mathbb{E}_{\boldsymbol{P}}\left[\sigma_{g}(\|\boldsymbol{P}\|) e^{-j 2 \pi\left\langle\boldsymbol{r}_{0}, \boldsymbol{P}\right\rangle} e^{j 2 \pi\langle\boldsymbol{r}, \boldsymbol{P}\rangle}\right], \quad \forall \boldsymbol{r} \in \mathbb{S}^{1},
$$

with $\alpha=\|g\|_{1}, \boldsymbol{r}_{0}=\left(\cos \theta_{0}, \sin \theta_{0}\right)$ and $\langle\cdot, \cdot\rangle$ the Cartesian inner product. An expression for the extended filter $\hat{\omega}_{e}$ follows directly by extending Eq. 9 , to $\mathbb{R}^{2}$ :

$$
\hat{\omega}_{e}(\boldsymbol{r})=\alpha \mathbb{E}_{\boldsymbol{P}}\left[\sigma_{g}(\|\boldsymbol{P}\|) e^{-j 2 \pi\left\langle\boldsymbol{r}_{0}, \boldsymbol{P}\right\rangle} e^{j 2 \pi\langle\boldsymbol{r}, \boldsymbol{P}\rangle}\right], \quad \forall \boldsymbol{r} \in \mathbb{R}^{2} .
$$

Notice that when $\alpha=1$ and $g \geq 0$, this can be seen as the Fourier transform of the density function $f_{b}$, modulated by an exponential $e^{-j 2 \pi\left\langle\boldsymbol{r}_{0}, \boldsymbol{P}\right\rangle}$. Leveraging the shifting property of the Fourier transform, we finally get

$$
\hat{\omega}_{e}\left(\boldsymbol{r}+\boldsymbol{r}_{\mathbf{0}}\right)=\mathbb{E}_{\boldsymbol{P}}\left[e^{j 2 \pi\langle\boldsymbol{r}, \boldsymbol{P}\rangle}\right]:=\varphi_{\boldsymbol{P}}(\boldsymbol{r}), \quad \forall \boldsymbol{r} \in \mathbb{R}^{2},
$$


which is the characteristic function $\varphi_{\boldsymbol{P}}: \mathbb{R}^{2} \rightarrow \mathbb{C}$ of the random vector $\boldsymbol{P}$.

In conclusion, in the specific case where $\alpha=1$ and $g \geq 0$, the extended filter $\hat{\omega}_{e}$ can be seen as the characteristic function of the random vector $\boldsymbol{P}$, centred around $\boldsymbol{r}_{0} \in \mathbb{S}^{1}$, the steering direction. Similarly, the target beamshape $\hat{\omega}$ can be seen as the characteristic function of the random vector $\boldsymbol{P}$, centred around $\boldsymbol{r}_{0} \in \mathbb{S}^{1}$, and evaluated on the circle.

\subsection{Empirical Beamshapes and Asymptotic Convergence}

Assume that for a target beamshape $\hat{\omega}$, with circularly symmetric extended filter $\hat{\omega}_{e}$, we computed the density beamforming function $f_{b}$ as described in Section 3.2 and sampled $N$ independent polar coordinates from this density

$$
\left\{\left(p_{i}, \phi_{i}\right)\right\}_{i=1, \ldots, N} \stackrel{i . i . d .}{\sim} f_{b} .
$$

If we place antennas at these random locations, and impose beamforming weights $w_{i}$ for each antenna, the resulting array will have a beamshape given by

$$
b_{N}(\theta)=\sum_{i=1}^{N} w_{i} e^{j 2 \pi p_{i} \cos \left(\theta-\phi_{i}\right)}, \quad \forall \theta \in[0,2 \pi] .
$$

Choosing the beamforming weights $w_{i}$ as

$$
w_{i}=\frac{\alpha}{N} \sigma_{g}\left(p_{i}\right) e^{-j 2 \pi p_{i} \cos \left(\phi_{i}-\theta_{0}\right)}, \quad i=1, \ldots, N,
$$

leads to the empirical beamshape, finite-sample version of Eq. 9]:

$$
b_{N}(\theta)=\frac{\alpha}{N} \sum_{i=1}^{N} \sigma_{g}\left(p_{i}\right) e^{-j 2 \pi p_{i} \cos \left(\phi_{i}-\theta_{0}\right)} e^{j 2 \pi p_{i} \cos \left(\theta-\phi_{i}\right)} .
$$

Notice that, since the density beamforming function $f_{b}$ is independent of the steering direction $\boldsymbol{r}_{0}=\left[\cos \left(\theta_{0}\right), \sin \left(\theta_{0}\right)\right]$, the layout of the array does not need to be changed in order to re-focus the beamshape towards another direction. For this, it is sufficient to update the beamforming weights in Eq. (11) with a new focus $\theta_{0}$.

Observe moreover that when $\sigma_{g}=1$, the beamforming weights $w_{i}$ in Eq. (11) are simply matched beamforming weights, which steer the beamshape around $\theta_{0}$. The empirical beamshape is an unbiased estimate of the target beamshape $\hat{\omega}$. Moreover, depending on the function $g$, this estimate can also be shown to be consistent. This follows from the uniform strong law of large numbers [13]:

Theorem 1 (Asymptotic Convergence of the Empirical beamshape). Assume an array formed by $N$ antennas placed at locations $\boldsymbol{P}_{i} \in$ $\mathbb{R}^{2}$, with polar coordinates $\left(p_{i}, \phi_{i}\right)$ randomly drawn from the density beamforming function $f_{b}$. Write further the resulting empirical beamshape of the array as

$$
b_{N}(\theta)=\frac{1}{N} \sum_{i=1}^{N} \psi\left(\boldsymbol{P}_{i}, \theta\right)
$$

where

$$
\psi\left(\boldsymbol{P}_{i}, \theta\right):=\alpha \sigma_{g}\left(p_{i}\right) e^{-j 2 \pi p_{i} \cos \left(\phi_{i}-\theta_{0}\right)} e^{j 2 \pi p_{i} \cos \left(\theta-\phi_{i}\right)} .
$$

Then, if the function $\psi(\cdot, \theta)$ is continuous and measurable for all $\theta \in[0,2 \pi]$

$$
\sup _{\theta \in[0,2 \pi]}\left\|b_{N}(\theta)-\hat{\omega}(\theta)\right\|_{2} \stackrel{a . s .}{\rightarrow} 0
$$

as the number of antennas $N$ grows to infinity.

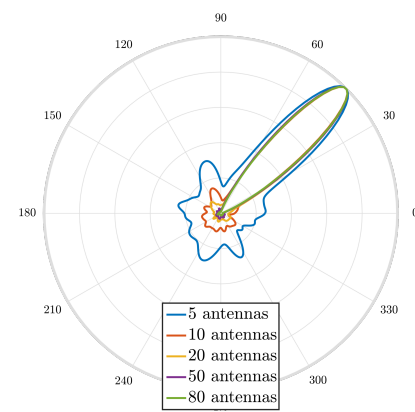

(a) Average beamshape with the number of antennas for the Gaussian beamshape.

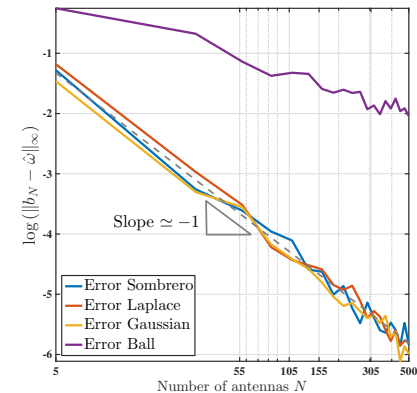

(b) Uniform error between the empirical beamshape and the target beamshape. Averaged over 10 experiments.
Fig. 1: Convergence analysis of the empirical beamshape to the target as the number of antennas increases for the 4 examples investigated in Section 4

A study of the function $\psi$ reveals that a sufficient condition for $b_{N}$ to be a consistent estimate of $\hat{\omega}$ is for the function $g$ to be either strictly positive or strictly negative. For functions $g$ that switch signs, we can still use the classical strong law of large numbers to show point-wise convergence. The empirical beamshapes for the Laplace, Sombrero and Gaussian functions, investigated in Section 4, converge extremely quickly, approximately as $1 / N$ (see Fig. 1).

\section{IMPORTANT LAYOUT DISTRIBUTIONS}

We first discuss the traditional case, targeting one individual point, before the case of targeting whole regions. Following the framework, for a given target $\hat{\omega}(\theta)$, we need to design an extended filter $\hat{\omega}_{e}(r, \theta)$ defined on the whole plane, and determine an appropriate distribution $f_{b}$ for achieving the target. The derived beamforming density functions are a consequence of known Hankel transform pairs (see Section 3.2).

\subsection{Focusing on a single point}

To target a single direction $\theta_{0}$, the most obvious extended filter is $\hat{\omega}_{e}(r, \theta)=\delta\left(\theta-\theta_{0}\right) \delta(r-1)$. The resulting beamforming function is $\omega(p, \phi)=e^{j 2 \pi p \cos \left(\theta_{0}-\phi\right)}$. This has unbounded support, and thus no beamforming density function exists. In practise, we have to sample over a finite disc, which is effectively achieved by an array that targets matched beamforming.

\subsubsection{Sombrero}

Consider the following $\hat{g}$, the Sombrero function,

$$
\hat{g}(r)=\sigma \frac{J_{1}\left(2 \pi \frac{r}{\sigma}\right)}{r},
$$

where $\sigma>0$. The beamforming density function is then, up to rescaling, a uniform distribution over the disc of radius $1 / \sigma$ :

$$
f_{b}(p, \phi) \propto \begin{cases}1 & \text { if } p \leq 1 / \sigma \\ 0 & \text { otherwise }\end{cases}
$$

From this we can conclude that for matched beamforming, a uniformly distributed array across a given area will get us close to the 
Sombrero function, which as the array diameter increases, converges to a Dirac. This observation has been made empirically in the past, and implemented as such in large arrays such as LOFAR.

\subsubsection{Radial Laplace}

Instead of trying to approximate a Dirac, it may be wiser to target a better behaved function, with an explicit control on the beamshape decay around the focus point $\theta_{0}$. This is achieved by the radial Laplace function: $\hat{g}(r)=e^{-\frac{r}{\sigma}}$ where $\sigma>0$. The beamforming density function is then, up to a rescaling function,

$$
f_{b}(p, \phi) \propto \frac{1}{\left(1+4 \pi^{2} \sigma^{2} p^{2}\right)^{\frac{3}{2}}} .
$$

Example Sombrero and radial Laplace beamshapes are shown in Figs. $2 a$ and $2 b$ The Laplace beamshape has almost no sidelobes, with its energy focused around the point of interest. In [14] we used this beamshape for the purpose of imaging by beamforming. By trading spatial resolution for smoother sidelobes, we could obtain an artifact-free image that is much easier to process.

\subsection{Targeting regions}

Flexibeam [6] presented two extended filters to target regions, the ball indicator function and the circularly symmetric Gaussian function. We now present the beamforming density functions associated to both these filters.

\subsubsection{Ball Indicator}

Consider the following disc of radius $R$ :

$$
\hat{g}(r)=\left\{\begin{array}{ll}
1, & r \leq R \\
0, & \text { otherwise }
\end{array} .\right.
$$

The beamforming density function is then, up to a rescaling function,

$$
f_{b}(p, \phi) \propto \frac{\left|J_{1}(2 \pi R p)\right|}{p} .
$$

It is interesting to note that, due to the Hankel pair relationship, approximating a region with the ball achieves a Sombrero beamforming density, while approximating a Sombrero requires a uniform density in a disc.

\subsubsection{Bi-dimensional Gaussian}

An alternative way to cover a region is to use a bi-dimensional Gaussian filter:

$$
\hat{g}(r)=\frac{1}{2 \pi \sigma^{2}} e^{-\frac{r^{2}}{2 \sigma^{2}}},
$$

where $\sigma>0$. The beamforming density function is then given by, up to a rescaling function

$$
f_{b}(p, \phi) \propto e^{-2 \pi^{2} \sigma^{2} p^{2}} .
$$

Example ball indicator and Gaussian beamshapes are shown in Figs. 2c and 2d The discontinuity of the ball indicator function breaks the requirements for Theorem 1 to be applicable. Hence, the beamshape demonstrates a significant side-lobe structure, also known as the Gibbs phenomenon. In contrast, the Gaussian is very smooth but does not isolate the portion of interest as well.
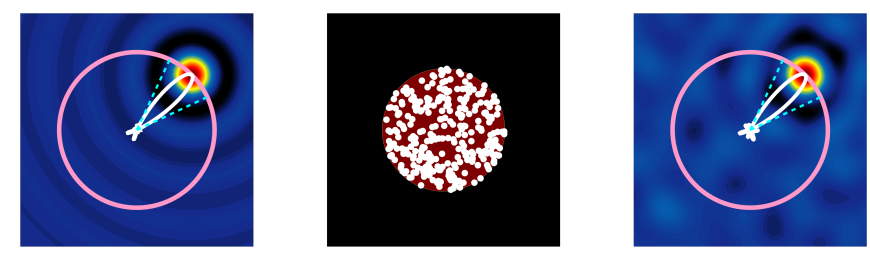

(a) Sombrero.
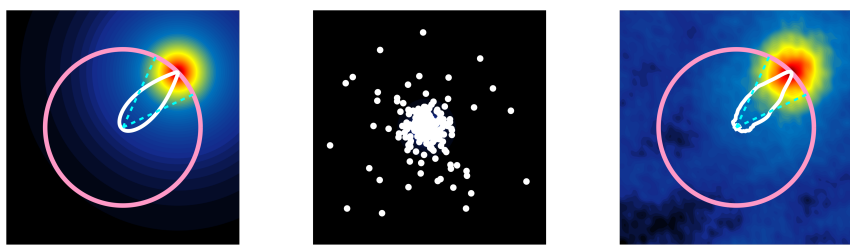

(b) Radial Laplace.
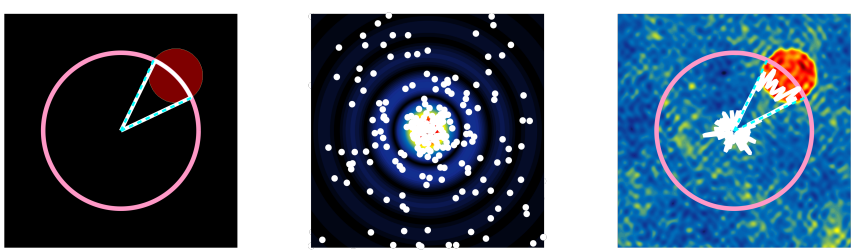

(c) Ball Indicator.
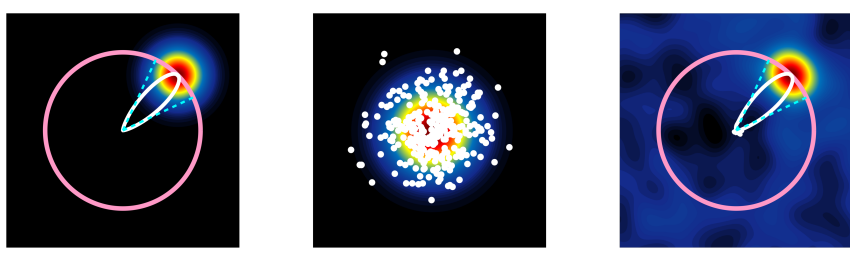

(d) Bi-dimensional Gaussian.

Fig. 2: Example beamshapes and layouts from their associated density. Left: target/extended filters, Middle: beamforming density function and random array layout, Right: empirical beamshape and extended filter.

We investigated the use of the Gaussian beamshape in [15], for the purpose of surveying large portions of the sky in radio astronomy. The resulting beamshape exhibited substantially more energy concentration within the region-of-interest than the state-of-the-art.

\section{CONCLUSIONS}

We noticed flexibeam, although powerful, gave no hints on where to place antennas. We thus brought the problem of antenna positioning into the framework by interpreting in a probabilistic setup, hence preserving the analytical scalable nature of the problem statement.

This led to a proof on the beamshape convergence, whose rate was further shown empirically to be as fast as $1 / N$. We show that matched beamforming weights used with inadequately designed layouts yield beamshapes far from targeting a single point. Indeed, used judiciously, those same weights can result in a sharply decaying narrow beamshape.

Future work includes a joint optimisation in both layout and beamforming weight, bridging flexarray and flexibeam, as well as extending the framework to $3 \mathrm{D}$ beamforming. 


\section{REFERENCES}

[1] D. K. Cheng, "Optimization techniques for antenna arrays," Proceedings of the IEEE, vol. 59, no. 12, pp. 1664-1674, 1971.

[2] R. J. Mailloux, Phased array antenna handbook. Artech House Boston, 2005, vol. 2.

[3] L. W. Schmerr Jr, Fundamentals of ultrasonic phased arrays. Springer, 2014, vol. 215.

[4] B. Rafaely, Fundamentals of spherical array processing. Springer, 2015, vol. 8.

[5] L. C. Godara, "Application of Antenna Arrays to Mobile Communications, Part II: Beam-forming and Direction-of-Arrival Considerations," Proceedings of the IEEE, vol. 85, no. 8, pp. 1195-1245, 1997.

[6] P. Hurley and M. Simeoni, "Beamforming towards regions of interest for multi-site mobile networks," in International Zurich Seminar on Communications, 2016, p. 94.

[7] P. Rocca, G. Oliveri, R. J. Mailloux, and A. Massa, "Unconventional Phased Array Architectures and Design Methodologies: A Review," Proceedings of the IEEE, vol. 104, no. 3, pp. 544-560, 2016.

[8] D. H. Johnson and D. E. Dudgeon, Array signal processing: concepts and techniques. Simon \& Schuster, 1992.

[9] S. Smith, G. Trahey, and O. Von Ramm, "Two-dimensional arrays for medical ultrasound," Ultrasonic Imaging, vol. 14, no. 3, pp. 213-233, 1992.

[10] M. Van Haarlem, M. Wise, A. Gunst, G. Heald, J. McKean, J. Hessels, A. De Bruyn, R. Nijboer, J. Swinbank, R. Fallows et al., "LOFAR: The low-frequency array," Astronomy \& Astrophysics, vol. 556, p. A2, 2013.

[11] A. R. Thompson, J. M. Moran, and G. W. Swenson Jr, Interferometry and synthesis in radio astronomy. John Wiley \& Sons, 2008.

[12] P. Hurley and M. Simeoni, "Flexibeam: analytic spatial filtering by beamforming," in International Conference on Acoustics, Speech and Signal Processing (ICASSP), IEEE, March 2016.

[13] V. Panaretos, Statistics for Mathematicians: A Rigorous First Course. Springer International Publishing, 2016.

[14] M. Simeoni and P. Hurley, "Laplace Beamshapes for PhasedArray Imaging," in International BASP Frontiers workshop 2017, 2016.

[15] P. Hurley and M. Simeoni, "On Flexibeam for radio interferometry," in International BASP Frontiers workshop 2017, 2016. 